\title{
Asthma is not associated with the need for surgery in Crohn's disease when controlling for smoking status: a population-based cohort study
}

This article was published in the following Dove Press journal:

Clinical Epidemiology

\section{Ellen Kuenzigl-3 \\ Mohsen Sadatsafavi ${ }^{4}$ \\ J Antonio Aviña-Zubieta ${ }^{5}$ \\ Rebecca M Burne ${ }^{6}$ \\ Michal Abrahamowicz ${ }^{6,7}$ \\ Marie-Eve Beauchamp ${ }^{7}$ \\ Gilaad G Kaplan ${ }^{8-11, *}$ \\ Eric I Benchimol ${ }^{1-3,12,13, *}$}

'Children's Hospital of Eastern Ontario (CHEO) Inflammatory Bowel Disease Centre, Division of Gastroenterology,

Hepatology and Nutrition, Children's

Hospital of Eastern Ontario, Ottawa, ON,

Canada; ${ }^{2}$ Health Information Technology

Program, CHEO Research Institute,

Ottawa, ON, Canada; ${ }^{3}$ Institute for Clinical

Evaluative Sciences, Ottawa, ON, Canada;

${ }^{4}$ Faculty of Pharmaceutical Sciences,

University of British Columbia, Vancouver,

BC, Canada; ${ }^{5}$ Arthritis Research Canada,

Division of Rheumatology, University of

British Columbia, Vancouver, BC, Canada;

${ }^{6}$ Department of Epidemiology, Biostatistics

and Occupational Health, McGill University,

Montreal, QC, Canada; ${ }^{7}$ Centre for Health

Outcomes Research (CORE), Research

Institute of the McGill University Health

Centre, Montreal, QC, Canada; ${ }^{8}$ Department of Medicine, University of Calgary, Calgary,

AB, Canada; ${ }^{9}$ Department of Community

Health Sciences, University of Calgary,

Calgary, AB, Canada; ${ }^{10} \mathrm{O}$ 'Brien Institute for

Public Health, University of Calgary, Calgary,

$A B$, Canada; "Snyder Institute for Chronic

Diseases, University of Calgary, Calgary,

AB, Canada; ${ }^{2}$ Department of Pediatrics,

University of Ottawa; Ottawa, ON, Canada;

${ }^{13}$ School of Epidemiology and Public Health,

University of Ottawa, Ottawa, ON, Canada

*These authors contributed equally to this work

Correspondence: M Ellen Kuenzig

Children's Hospital of Eastern Ontario, 401

Smyth Road, Ottawa, ON, KIH 8LI, Canada

Tel +l (6I3) 7985555 (ext I0308)

$\mathrm{Fax}+\mathrm{I}(613) 7384854$

Email mkuenzig@cheo.on.ca
Purpose: Growing evidence suggests asthma and Crohn's disease commonly cooccur. However, the impact of asthma on the prognosis of Crohn's disease is unknown. The aim of our study was to assess the effect of asthma on the need for intestinal resection in patients with Crohn's disease while adjusting for smoking status, imputed from a smaller, secondary data set.

Patients and methods: Using health administrative data from a universally funded healthcare plan in Alberta, Canada, we conducted a cohort study to assess the effect of asthma on the need for surgery in patients with Crohn's disease diagnosed between 2002 and $2008(\mathrm{~N}=2,113)$. Validated algorithms were used to identify incident cases of Crohn's disease, cooccurring asthma, and intestinal resection. The association between asthma and intestinal resection was estimated using multivariable Cox proportional hazards regression. Smoking status was imputed using a novel method using martingale residuals, derived from a data set of 485 patients enrolled in the Alberta Inflammatory Bowel Disease Consortium (2007 to 2014) who completed environmental questionnaires. All analyses were adjusted for age, sex, rural/urban status, and mean neighborhood income quintile.

Results: Asthma did not increase the risk of surgery in the health administrative data when not adjusting for smoking status (HR 1.03, 95\% CI 0.81 to 1.29 ). The association remained nonsignificant after imputing smoking status in the health administrative data (HR 1.03, 95\% CI 0.81 to 1.29 ).

Conclusion: Although asthma is associated with an increased risk of Crohn's disease, cooccurring asthma is not associated with the risk of surgery in these patients. This null association persisted after adjusting for smoking status. This study described a novel method to adjust for confounding (smoking status) in time-to-event analyses, even when the confounding variable is unmeasured in health administrative data.

Keywords: Crohn's disease, asthma, surgery, unmeasured confounding, health administrative data

\section{Introduction}

Crohn's disease is a type of inflammatory bowel disease (IBD) that can cause inflammation throughout the gastrointestinal tract. Both asthma and Crohn's disease are immune-mediated diseases that are becoming increasingly common, ${ }^{1-3}$ and there is growing evidence that these two diseases often cooccur. Studies evaluating the association between these two diseases have estimated that odds of asthma among people with Crohn's disease are $\sim 1.5$-fold higher than the odds of asthma among people without Crohn's disease. ${ }^{4-6}$ The reasons for this co-occurrence may be shared 
common characteristics, including their underlying pathogenesis, as well as environmental and genetic risk factors. The impact of co-existing immune-mediated conditions on the prognosis of Crohn's disease is not well understood and may vary across conditions. An increased risk of surgery has been observed in Crohn's disease patients with at least one other immune-mediated disease. ${ }^{7}$ Although asthma was the most common comorbidity among patients included in this study, the specific impact of asthma on the risk of intestinal resection in Crohn's disease remains unknown. ${ }^{7,8}$

Nearly, half of all patients with Crohn's disease will require an intestinal resection within 10 years of their diagnosis; however, the risk of surgery has decreased over the past half century. ${ }^{9}$ Current tobacco smoking is the most consistently replicated environmental risk factor for surgery in Crohn's disease. ${ }^{10}$ Smoking is also associated with an increased risk of asthma. ${ }^{11}$ However, patients' smoking history is not readily available in health administrative data, and an inability to adjust for smoking is a common limitation of epidemiological studies using health administrative data to assess outcomes for which smoking is a risk factor. This includes studies that aim to evaluate the risk of surgery in patients with Crohn's disease. ${ }^{12-14}$ Despite this limitation, health administrative data are important tools for the better understanding of risk factors responsible for the development and outcomes of Crohn's disease.

Until recently, the availability of methods to impute information on unmeasured confounders in time-to-event analyses has been limited. Burne and Abrahamowicz ${ }^{15}$ developed a method, applicable to multivariable analyses with Cox proportional hazards models, to impute unmeasured confounders using martingale residuals. In this method, imputation of confounding variables not available in health administrative data requires that these unmeasured confounding variables be available in a validation data set, either a subset of the health administrative data or an external data set. In simulations, this method provided more accurate results in adjusting for an unmeasured confounder than other methods applicable to time-to-event analyses. ${ }^{15}$ Thus, the objective of our study was to evaluate the impact of asthma on the need for an intestinal resection in patients with Crohn's disease, while adjusting for smoking status, despite the limitation of smoking status being unmeasured in the health administrative data. We did this by using a validation data set in which smoking status was measured and applying the martingale residual-based imputation method. ${ }^{15}$

\section{Patients and methods}

\section{Study design and data sources}

Health administrative data

This study was approved by the Conjoint Research Ethics Board at the University of Calgary. We conducted a population-based, retrospective cohort study to examine the association between asthma and the need for surgery in individuals living with Crohn's disease using deidentified health administrative data from the Alberta Health Care Insurance Plan, a universally funded healthcare plan in the Western Canadian province of Alberta. All data were obtained from Alberta Health and Wellness and have been previously described., ${ }^{4,16,17}$ For all legal residents of Alberta, Canada ( $>99 \%$ of the population), the data include demographic and geographic information (Registry database), outpatient claims made by all Alberta physicians in the delivery of health care (Alberta Health Physician Claims database), hospitalizations (Discharge Abstract Database), and hospital-based ambulatory care such as emergency department encounters and outpatient procedures (Ambulatory Care Classification System). Patients are assigned a unique personal health identification number, which was used to link deterministically between data sources and follow patients through time. Databases were available from April 1, 1994 to March 31, 2010; the Ambulatory Care Classification System began from April 1, 1997.

\section{Identifying incident cases of Crohn's disease}

A previously validated algorithm was used to identify all cases of IBD based on International Classification of Diseases (ICD)-9 (Crohn's disease: 555; ulcerative colitis: 556) and ICD-10 (Crohn's disease: K50, ulcerative colitis: K51) codes. ${ }^{17} \mathrm{~A}$ detailed description of the algorithm is provided in Table S1. A validated scoring system based on the relative number of diagnostic codes for ulcerative colitis or Crohn's disease was used to confirm type of IBD with a specificity of $>99 \%$ and sensitivity of $94 \%$ for the diagnosis of Crohn's disease. ${ }^{17}$ Individuals with ulcerative colitis and in whom the type of IBD could not be determined based on this scoring system were excluded (Figure 1).

We differentiated between incident and prevalent cases by applying a previously validated washout period. For individuals $\geq 10$ years of age at Crohn's disease diagnosis, a washout period of 8 years was required. For individuals $<10$ years of age at diagnosis with Crohn's disease, a 3-year washout period was required. ${ }^{17,18}$ Patients diagnosed with Crohn's disease 


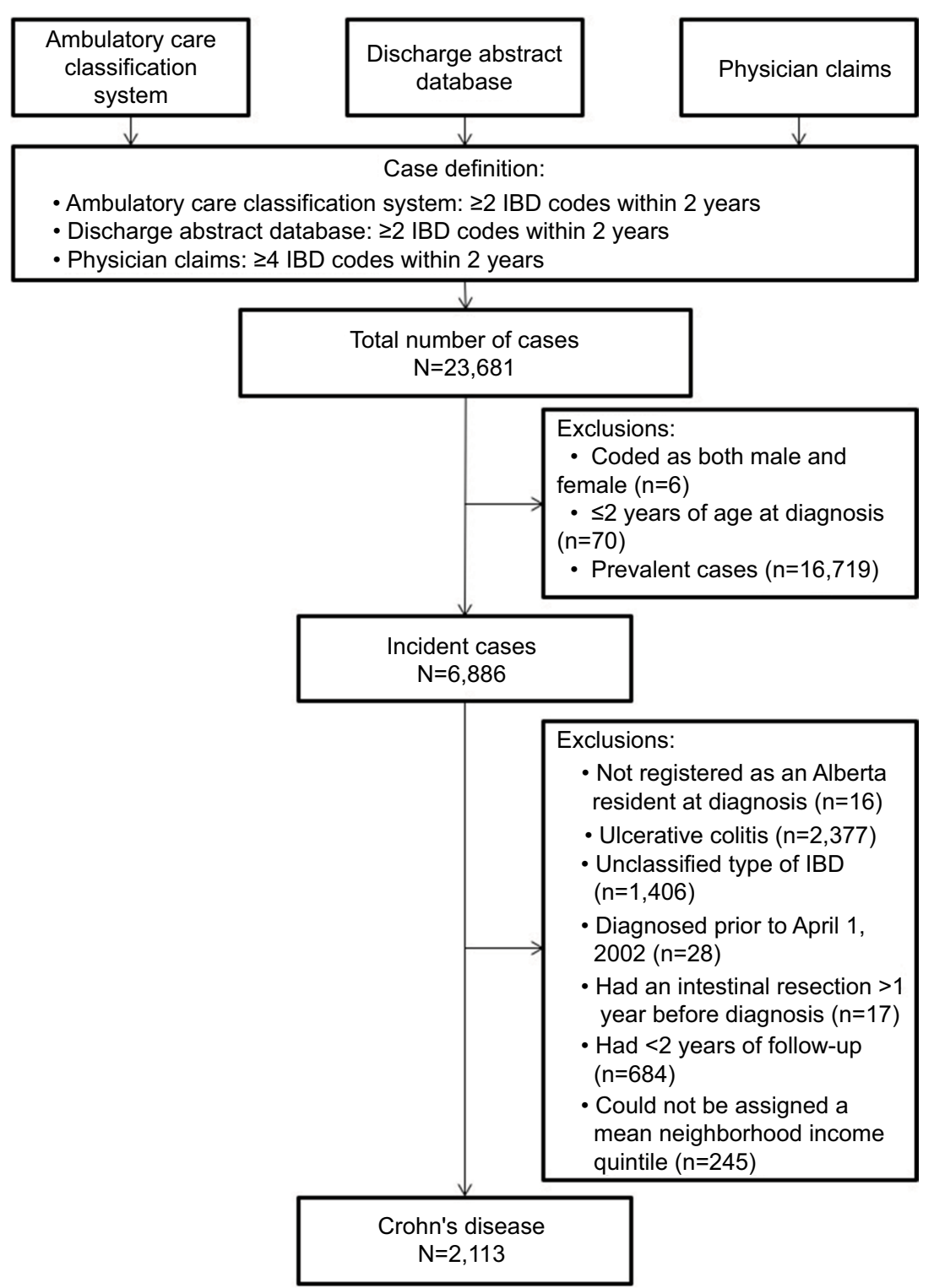

Figure I Flow diagram for the identification of incident cases of Crohn's disease in the health administrative data.

Abbreviation: IBD, inflammatory bowel disease.

prior to 3 years of age were excluded to minimize the risk of misclassification bias arising from challenges in diagnosing asthma in young children. The date of Crohn's disease diagnosis was the date of the first healthcare contact with a code for IBD. Patients were followed from the date of diagnosis until either undergoing intestinal resection or being censored (death, emigration out of Alberta, or the end of the study). Only patients diagnosed prior to April 1, 2008 were included to ensure all patients had at least 2 years of follow-up.

\section{Cooccurring asthma}

Prevalent cases of asthma were identified using an algorithm previously validated in the province of Ontario, Canada, using ICD-9 code 493 and ICD-10 code $\mathrm{J} 45 .{ }^{19}$ The algorithm is described in Table S1. The diagnosis of asthma could either precede or follow the diagnosis of IBD.

\section{Intestinal resection}

Individuals undergoing intestinal resection were identified from the Discharge Abstract Database using a previously validated list of ICD-10 procedural codes for intestinal resection (Table S2). ${ }^{14}$ Only the first occurrence of surgery was included. Surgeries occurring during the year prior to diagnosis with Crohn's disease were assumed to have occurred at the time of diagnosis. If surgery occurred more than a year prior to diagnosis, the patient was excluded from the analysis $(n=17)$. We excluded all individuals diagnosed with Crohn's disease prior to April 1, 2002, as this was when Alberta Health 
and Wellness began using ICD-10 procedural codes in the Discharge Abstract Database. Procedural codes used prior to April 1, 2002 have not been validated previously and were therefore not used in this study. We conducted a sensitivity analysis to exclude surgeries performed in the year prior to and the month following the date of diagnosis.

\section{Validation data set:Alberta IBD Consortium}

The validation data (including information on smoking status of patients) was derived from patients enrolled in the Alberta IBD Consortium, recruited from an IBD clinic in Calgary, Alberta, Canada, between 2007 and 2014 (Figure 2). All patients $(\mathrm{N}=509)$ included in the cohort have undergone extensive chart review that includes information on 1) their date of diagnosis; 2) the number and dates of any intestinal resection(s); and 3) coexisting conditions (including asthma). In addition, patients completed an environmental questionnaire that included information on their history of cigarette smoking, including when they started and stopped smoking. The environmental questionnaire was completed in most patients $(n=504)$. If it was not $(n=5)$, smoking status was ascertained from their medical chart.

Current smokers had smoked at least one cigarette per day for at least 1 year prior to being diagnosed with Crohn's disease. Nonsmokers had quit smoking at least one year prior to diagnosis or were lifelong nonsmokers. Smoking status was assumed to be constant over the course of the study. We conducted sensitivity analyses in which the definition of smoking varied: 1) nonsmokers had quit smoking for at least 3 years prior to diagnosis and smokers had smoked for at least 3 years and 2) nonsmokers had quit smoking for at least 5 years prior to diagnosis and smokers had smoked for at least 5 years. Previous studies have shown retrospective recall of smoking habits to be valid with a sensitivity and specificity of $92 \%$ and $98 \%$, respectively. ${ }^{20}$ Characteristics of patients in the health administrative data and the validation sample are compared in Table 1 and Figure S1.

\section{Measured confounding variables}

Mean neighborhood income was assigned using the Postal Code Conversion File Plus provided by Statistics Canada. ${ }^{21}$ Census metropolitan areas and metropolitan influence zones were used to determine if a person was living in an urban environment. $^{22}$ Individuals living in rural environments but with a high degree of interaction with urban environments (eg, by commuting from a rural to urban area for employment) were considered to live in an urban environment. Mean neighborhood income quintile and rural/urban status

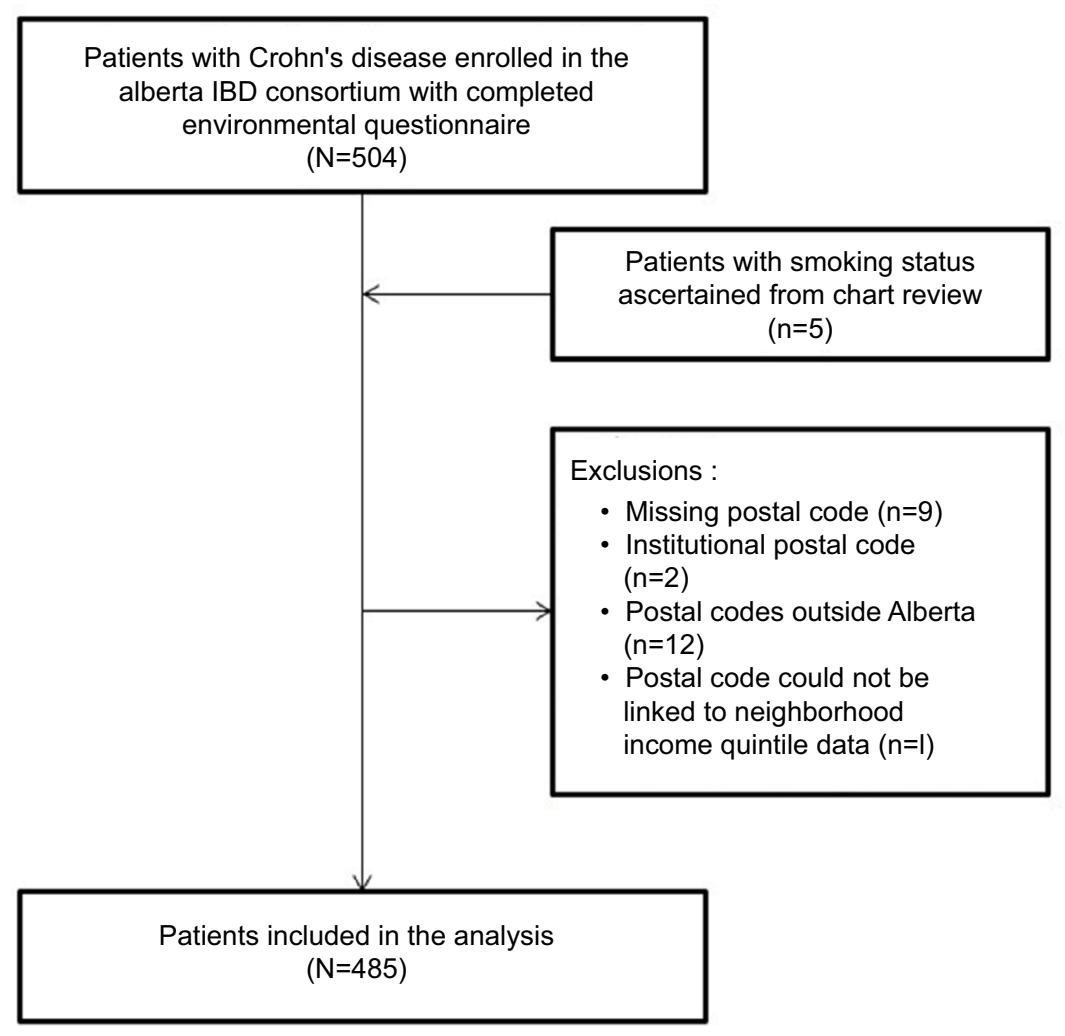

Figure 2 Flow diagram for the identification of cases of Crohn's disease enrolled in the Alberta IBD Consortium. Abbreviation: IBD, inflammatory bowel disease. 
Table I Comparison of the characteristics of Crohn's disease patients included in the health administrative data and the Alberta IBD Consortium data

\begin{tabular}{|c|c|c|}
\hline Characteristic & $\begin{array}{l}\text { Health administrative } \\
\text { data }(\mathrm{N}=2,1 \mid 3)\end{array}$ & $\begin{array}{l}\text { Alberta IBD } \\
\text { Consortium } \\
(\mathbf{N}=485)\end{array}$ \\
\hline \multicolumn{3}{|c|}{ Age at Crohn's disease diagnosis } \\
\hline $\mathrm{Al}: \leq 16$ years, $\mathrm{n}(\%)$ & $210(9.9)$ & $67(13.8)$ \\
\hline A2: $17-40$ years, $n(\%)$ & $\mathrm{I}, 010(47.8)$ & $336(69.3)$ \\
\hline A3: >40 years, $n(\%)$ & $893(42.3)$ & $82(16.9)$ \\
\hline Mean (SD) & $37.6(17.7)$ & $28.3(12.9)$ \\
\hline Median (IQR) & $36(27)$ & $25(16)$ \\
\hline \multicolumn{3}{|l|}{ Asthma, n (\%) } \\
\hline Yes & $337(15.9)$ & $24(4.9)$ \\
\hline No & I,776 (84.I) & $46 I(95.1)$ \\
\hline \multicolumn{3}{|l|}{ Surgery, n (\%) } \\
\hline Yes & $532(25.2)$ & $244(50.3)$ \\
\hline No & $|58|(74.8)$ & $24 I(49.7)$ \\
\hline \multicolumn{3}{|l|}{ Sex, n (\%) } \\
\hline Female & I, $186(56.1)$ & $286(59.0)$ \\
\hline Male & $927(43.9)$ & $199(41.0)$ \\
\hline \multicolumn{3}{|c|}{ Rural/urban residence, $\mathbf{n}(\%)$} \\
\hline Rural & $520(24.6)$ & $58(12.0)$ \\
\hline Urban & I,593 (75.4) & $427(88.0)$ \\
\hline \multicolumn{3}{|c|}{ Mean neighborhood income quintile, $n$ (\%) } \\
\hline QI (lowest) & $450(21.3)$ & $61(12.6)$ \\
\hline Q2 & $503(23.8)$ & $91(18.8)$ \\
\hline Q3 & $413(19.5)$ & $111(22.9)$ \\
\hline Q4 & $389(18.4)$ & $113(23.3)$ \\
\hline Q5 (highest) & $358(16.9)$ & $109(22.5)$ \\
\hline \multicolumn{3}{|c|}{ Duration of follow-up, years } \\
\hline Mean (SD) & $5.0(1.8)$ & $14.8(10.6)$ \\
\hline Median (IQR) & $5.0(3.1)$ & $12.1(14.5)$ \\
\hline Range & 2 to 8 & 0.24 to 46.9 \\
\hline \multicolumn{3}{|c|}{ Smoking status at diagnosis, n (\%) } \\
\hline Current smoker & $\mathrm{n} / \mathrm{a}$ & $135(27.8)$ \\
\hline Nonsmoker & $\mathrm{n} / \mathrm{a}$ & $350(72.2)$ \\
\hline
\end{tabular}

Abbreviations: IBD, inflammatory bowel disease; IQR, interquartile range; $n / a$, not applicable.

were determined at the time of diagnosis of Crohn's disease in the health administrative data and at time of study enrollment in the validation data and did not vary over the course of disease. Patients in the health administrative data living in a dissemination area that could not be linked to rural/urban status or neighborhood income were excluded $(n=245)$. In addition, 24 patients in the validation data with a missing postal code, postal code outside Alberta, or a postal code that could not be linked to rural/urban status or neighborhood income were excluded.

\section{Statistical analysis}

The proportion of patients with Crohn's disease with and without asthma were compared for the following characteristics: age at diagnosis of Crohn's disease, as defined a priori by the Montreal Classification (A1: $\leq 16$ years; A2: $17-40$ years; and A3: $>40$ years of age at diagnosis $)^{23}$, sex, mean neighborhood income quintile, rural/urban status, and the need for intestinal resection. The mean (SD) and median (interquartile range [IQR]) age at diagnosis and duration of follow-up were compared for individuals with and without asthma. In addition, we compared the characteristics of patients included in the health administrative data and validation data.

Kaplan-Meier analyses were used to assess the impact of asthma on the time to surgery in patients with Crohn's disease. We used a multivariable Cox proportional hazards model to evaluate the independent association between asthma and surgery in patients with Crohn's disease, adjusting for confounders measured in the health administrative data (age at Crohn's disease diagnosis - continuous with a quadratic term, sex, mean neighborhood income quintile, and living in an urban or rural environment). We then fit a second multivariable Cox proportional hazards model including the confounders measured in the health administrative data and the imputed smoking status. We conducted a sensitivity analysis in which patients undergoing surgery in the year prior to diagnosis or 30 days after diagnosis were excluded from the analysis $(n=178$ in the health administrative data; $\mathrm{n}=39$ in the validation data).

\section{Imputation of smoking status}

Smoking status at the time of Crohn's disease diagnosis was imputed using a novel method based on martingale residuals, ${ }^{24}$ designed specifically to impute unmeasured confounders in Cox proportional hazards regression analyses. ${ }^{15}$ This method incorporates information on both the follow-up time and the patient's status (surgery or censoring) at the end of follow-up, to quantify the discrepancies between outcomes observed for individual subjects and their outcomes predicted from the model that accounts only for confounders measured in the health administrative data. The second step of the analysis uses the validation subsample to assess how the value of each confounder not measured in the health administrative data (smoking status) depends on the 1) martingale residuals; 2) exposure; and 3) confounders available in both the health administrative data and validation data. ${ }^{15}$ At the third step, the values of these additional confounders are imputed for all subjects in the health administrative data, based on the relationships estimated in the second step.

To implement this method, first, martingale residuals were estimated from Cox proportional hazards models run in 
the health administrative data and the validation data (these models included asthma and confounders measured in the health administrative data only). In addition to the exposure and measured confounders, the martingale residuals were used in a logistic regression model, fit in the validation data, with smoking status (our unmeasured confounder) as the binary outcome. The predicted probability of being a current smoker or nonsmoker from the logistic regression model was then used to impute smoking status for all subjects in the health administrative data. Then, the final Cox proportional hazards model incorporating asthma, measured confounders, and imputed smoking status were fit in the health administrative data.

In addition, we used an array approach to estimate the degree of bias and the impact of unmeasured smoking on the association between asthma and surgery. ${ }^{25}$ This method relies on estimates of the association between smoking and surgery, as well as the prevalence of smoking in people with and without asthma. The association between smoking and intestinal resection was derived from a meta-analysis (HR $1.27,95 \%$ CI 1.08 to 1.30$).{ }^{10}$ The prevalence of smoking in people with and without asthma was derived from the validation data and was allowed to vary.

All statistical analyses were conducted using $\mathrm{R}$ version 3.4.1 ${ }^{26}$ survival analysis was conducted using the survival package and Kaplan-Meier curves were created using the survminer package. ${ }^{27,28}$ Code to apply the martingale residualbased imputation method is available at https://github.com/ RMBurne/MR-based-imputation-use.

\section{Results}

There were 2,113 individuals in the Alberta health administrative data diagnosed with Crohn's disease after April 1, 2002 and had at least 2 years of follow-up following the diagnosis of Crohn's disease (Figure 1). The median duration of followup was 5.0 (IQR 1.8) years and was similar for people with and without asthma. Overall, 15.9\% $(337 / 2,113)$ had asthma and $25.1 \%(532 / 2,113)$ required an intestinal resection. Characteristics of Crohn's disease patients with and without asthma are presented in Table 2.

A total of 485 patients from the Alberta IBD Consortium were included in the validation data set (Figure 2). Of these, $24(4.9 \%)$ had asthma and the median duration of followup was 12.1 (IQR 14.5) years. An intestinal resection was required by $50.3 \%(244 / 485)$ of patients. The median time to surgery was 12.01 years (95\% CI 9.98 to 14.75$)$. There were no significant differences when comparing patients with and without asthma (Table S3). Patients in the validation data
Table 2 Characteristics of patients with Crohn's disease in health administrative data from Alberta, Canada

\begin{tabular}{|c|c|c|c|}
\hline Characteristic & $\begin{array}{l}\text { Total } \\
(\mathrm{N}=2,1 \mid 3)\end{array}$ & $\begin{array}{l}\text { Asthma } \\
(n=337)\end{array}$ & $\begin{array}{l}\text { No asthma } \\
(n=I, 776)\end{array}$ \\
\hline \multicolumn{4}{|l|}{ Surgery, n (\%) } \\
\hline Yes & $532(25.2)$ & $86(25.5)$ & $446(25.1)$ \\
\hline No & $\mathrm{I}, 58 \mathrm{I}(74.8)$ & $25 I(74.5)$ & $1330(74.9)$ \\
\hline \multicolumn{4}{|l|}{ Age at IBD diagnosis } \\
\hline $\mathrm{Al}: \leq 16$ years, $\mathrm{n}(\%)$ & $210(9.9)$ & 40 (II.9) & $170(9.6)$ \\
\hline A2: 17-40 years, $n(\%)$ & $1,010(47.8)$ & $175(5 \mid .9)$ & $835(47.0)$ \\
\hline A3: >40 years, $n(\%)$ & $893(42.3)$ & $122(36.2)$ & $771(43.4)$ \\
\hline Mean (SD), years & $37.6(17.7)$ & $36.0(19.0)$ & $37.9(17.4)$ \\
\hline Median (IQR), years & $36(27)$ & $3 I(49-2 I)$ & $37(50-23)$ \\
\hline \multicolumn{4}{|l|}{ Sex, n (\%) } \\
\hline Female & $\mathrm{I}, 186(56.1)$ & $217(64.4)$ & $969(54.6)$ \\
\hline Male & $927(43.9)$ & $120(35.6)$ & $807(45.4)$ \\
\hline \multicolumn{4}{|c|}{ Rural/urban residence, $\mathbf{n}(\%)$} \\
\hline Rural & $520(24.6)$ & $7 I(2 I .1)$ & $449(25.3)$ \\
\hline Urban & $1,593(75.4)$ & $266(78.9)$ & $\mathrm{I}, 327(74.7)$ \\
\hline \multicolumn{4}{|c|}{ Mean neighborhood income quintile, $n$ (\%) } \\
\hline QI (lowest) & $450(21.3)$ & $86(25.5)$ & $364(20.5)$ \\
\hline Q2 & $503(23.8)$ & $77(22.8)$ & $426(24.0)$ \\
\hline Q3 & $413(19.5)$ & $70(20.8)$ & $343(19.3)$ \\
\hline Q4 & $389(18.4)$ & $63(18.7)$ & $326(18.4)$ \\
\hline Q5 (highest) & $358(16.9)$ & $4 I(12.2)$ & $317(17.8)$ \\
\hline \multicolumn{4}{|c|}{ Duration of follow-up, years } \\
\hline Mean (SD) & $5.0(1.8)$ & $5.0(1.8)$ & $5.0(1.8)$ \\
\hline Median (IQR) & $5.0(3.1)$ & $5.1(3.2)$ & $5.0(3.1)$ \\
\hline Range & 2.0 to 8.0 & 2.0 to 8.0 & 2.0 to 8.0 \\
\hline
\end{tabular}

Abbreviations: IQR, interquartile range; IBD, inflammatory bowel disease.

were younger at diagnosis, and had a longer disease duration compared with patients in the health administrative data (Table 1; Figure S1).

\section{Association between asthma and intestinal resection}

Asthma was not associated with the risk of intestinal resection in either the health administrative data (crude HR 1.02, 95\% CI 0.81 to 129; Figure 3) or the validation data set (crude HR 0.82 , 95\% CI 0.45 to 1.50; Figure S2). These findings were consistent in both the health administrative data and validation data sets after adjusting for age, sex, mean neighborhood income quintile, and rural/urban status, but not for smoking status (Tables 3 and $\underline{\mathrm{S}} 4$, respectively). The association remained statistically nonsignificant when applying the martingale residual-based method to adjust for smoking status at diagnosis in the health administrative data (HR 1.01, 95\% CI 0.80 to 1.28; Table 3). Findings were unchanged with alternate smoking definitions (Table S5) and after excluding patients undergoing intestinal resection in the year prior to or the 30 days following IBD diagnosis (Table S6). Using the array approach and an assumption that $30 \%$ of people without asthma were current smokers, the adjusted relative risk ranged 

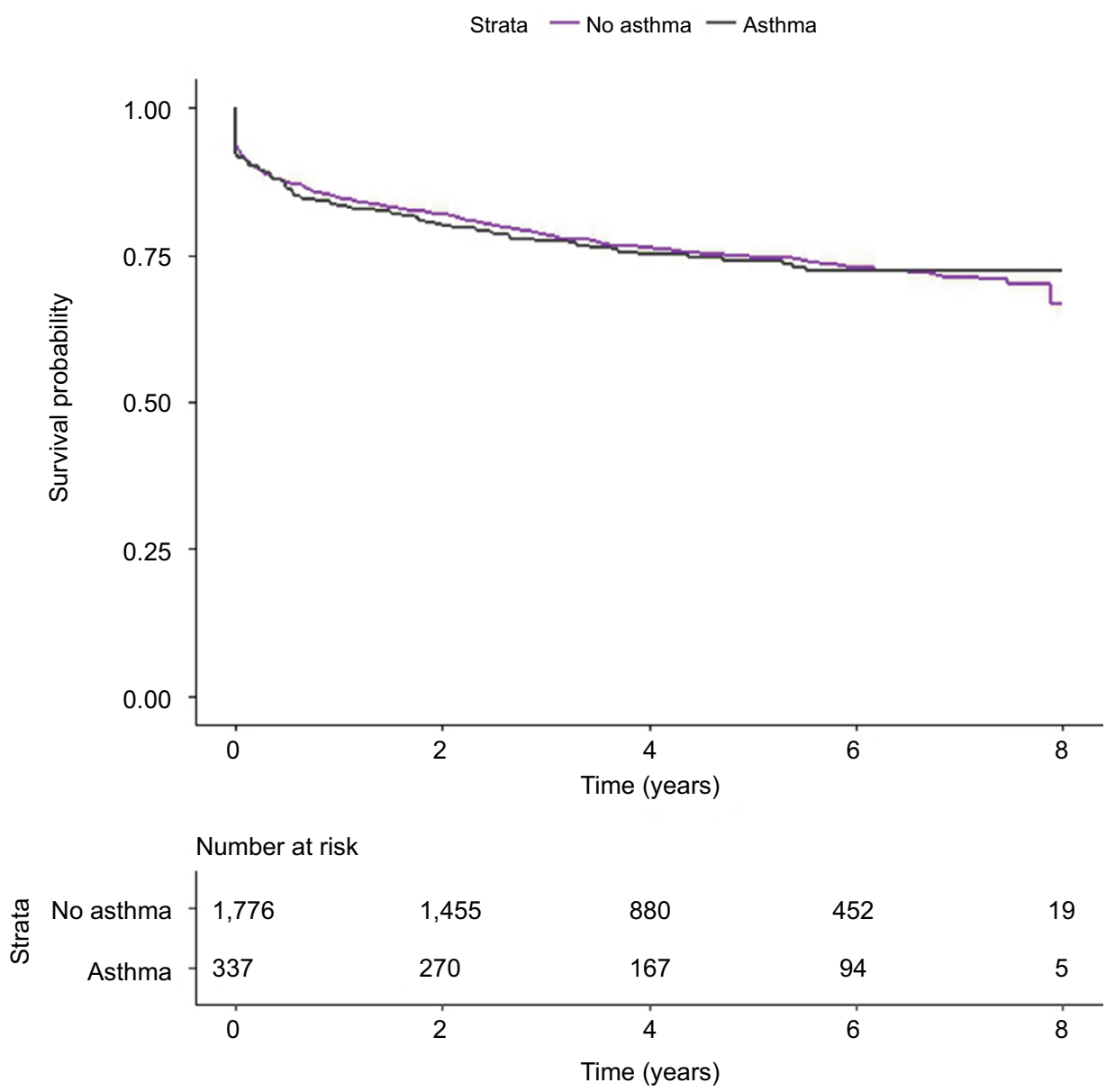

Figure 3 Kaplan-Meier survival curves depicting the time to surgery in patients with Crohn's disease with and without cooccurring asthma in health administrative data.

from 0.9 to 1.1 (Figure $\mathrm{S} 3$ ). Results were similar with differing rates of smoking among people without asthma (igure S3 $)$.

Smoking was not associated with the risk of intestinal resection in the validation sample (HR $1.14,95 \%$ CI 0.85 to 1.51). Smoking was associated with a higher risk of surgery in the model in which it was imputed (HR 1.31, 95\% CI 1.07 to 1.59$)$.

\section{Discussion}

Although asthma and Crohn's disease commonly cooccur, ${ }^{4-6}$ we did not find any difference in the risk of surgery among Crohn's disease patients with and without cooccurring asthma. These findings remained consistent after using a martingale residual-based method to impute smoking status in the health administrative data from a secondary data set that included smoking status. ${ }^{15}$

Extraintestinal manifestations of IBD have been linked to a more severe disease course, as measured by disease activity indices and an increased need for steroids and immunosuppression. ${ }^{29-32}$ However, the impact of extraintestinal manifestations on the need for surgery in patients with Crohn's disease is less clear. The presence of at least one extraintestinal manifestation does not impact the risk of surgery, while having at least one other comorbid immune-mediated disease is associated with an increased risk of surgery. ${ }^{7,30}$ The impact of extraintestinal manifestations on the prognosis of Crohn's disease may depend on the type of extraintestinal manifestation. For example, type I (pauciarticular) arthropathies are associated with a decreased risk of surgery, while type II (polyarticular) arthropathies are not associated with surgical risk. ${ }^{33}$ To the best of our knowledge, this is the first study to evaluate the impact of asthma on the risk of surgery in Crohn's disease patients.

Canadian health administrative data provide large sample sizes and population-based data free of selection bias due to the single-payer universal health care system. However, unmeasured confounding is an important limitation of studies conducted using health administrative data. This includes studies using health administrative data to identify factors associated with the risk of intestinal resection in patients with Crohn's disease despite smoking being the best documented risk factor 
Table 3 Cox proportional hazards model for the association between asthma and the need for surgery in patients with Crohn's disease in health administrative data from Alberta, Canada

\begin{tabular}{|c|c|c|}
\hline \multirow[t]{2}{*}{ Characteristic } & \multirow{2}{*}{$\begin{array}{l}\text { Health administrative } \\
\text { data only } \\
\text { HR }(95 \% \mathrm{Cl}) \\
\end{array}$} & \multirow{2}{*}{$\begin{array}{l}\begin{array}{l}\text { Model with imputed } \\
\text { smoking status }\end{array} \\
\text { HR }(95 \% \mathrm{Cl})\end{array}$} \\
\hline & & \\
\hline \multicolumn{3}{|l|}{ Asthma } \\
\hline Yes & $\mathrm{I} .03(0.8 \mathrm{I}$ to I.29) & I.0I (0.80 to I.28) \\
\hline No & REF & REF \\
\hline \multicolumn{3}{|c|}{ Age at Crohn's disease diagnosis } \\
\hline Linear term & $1.00(0.98$ to 1.02$)$ & $1.00(0.98$ to 1.02$)$ \\
\hline Quadratic term & 1.00 (1.00 to 1.00$)$ & $1.00(1.00,1.00)$ \\
\hline \multicolumn{3}{|l|}{ Sex } \\
\hline Female & $0.90(0.76$ to 1.07$)$ & $0.88(0.74$ to 1.05$)$ \\
\hline Male & REF & REF \\
\hline \multicolumn{3}{|c|}{ Rural/urban residence } \\
\hline Rural & $\mathrm{I} .07$ (0.88 to I.29) & $\mathrm{I} .03$ (0.84 to I.25) \\
\hline Urban & REF & REF \\
\hline \multicolumn{3}{|c|}{ Mean neighborhood income quintile } \\
\hline QI (lowest) & REF & REF \\
\hline Q2 & $0.75(0.58$ to 0.95$)$ & $0.75(0.59$ to 0.96$)$ \\
\hline Q3 & $0.70(0.54$ to 0.91$)$ & $0.72(0.55$ to 0.94$)$ \\
\hline Q4 & $0.86(0.66$ to $\mathrm{I} . \mathrm{II})$ & $0.86(0.66$ to 1.11$)$ \\
\hline Q5 (highest) & $0.74(0.57$ to 0.98$)$ & $0.77(0.59$ to 1.01$)$ \\
\hline \multicolumn{3}{|c|}{ Smoking status at diagnosis } \\
\hline Current smoker & $\mathrm{n} / \mathrm{a}$ & I.3I (I.07 to I.59) \\
\hline Nonsmoker & $\mathrm{n} / \mathrm{a}$ & REF \\
\hline
\end{tabular}

Abbreviations: HR, hazard ratio; REF, reference; n/a, not applicable.

for surgery in these patients. ${ }^{10,34}$ We demonstrated that it was feasible to adjust for smoking status, even if it is unmeasured in the health administrative data, by using a secondary data set that includes information on patients' smoking habits to impute smoking status in health administrative data. ${ }^{15}$ Results were similar when using an array approach to evaluate the degree to which unmeasured smoking may influence the association between asthma and surgery. ${ }^{25}$ Because the martingale residual-based method is currently not applicable to categorical unmeasured confounders with three or more categories, smoking status was included as a dichotomous variable (ie, current smokers were compared with current nonsmokers) and more nuanced metrics such as pack-years were not evaluated. However, the risk of intestinal resection in patients who quit smoking decreases over time, so that long-term risk of surgery in ex-smokers mirrors that of non-smokers. ${ }^{35,36}$ Thus, our findings may have been biased by not incorporating changes in smoking habits after the diagnosis of Crohn's disease. Further, measured confounders (rural/urban residence and mean neighborhood income quintile) were assumed to be constant over the course of this study, and bias may have been introduced if patients were to move between rural and urban communities or to neighborhoods with differing income levels.
Several limitations to our study exist. The results of our study may have been biased by our use of a validation data set derived from patients recruited from a tertiary IBD clinic. These patients may have been more likely to have more severe disease compared with the population-based health administrative data. Although we are unable to ascertain the severity of disease in the health administrative data, surgery is only performed in patients with complicated or medically refractory Crohn's disease. Patients in our validation data set had a much higher risk of surgery $(50.3 \%)$ compared with patients identified in the health administrative data (25.2\%). However, the difference in the overall risk of surgery may also be partially explained by the longer duration of followup among patients in the validation data arising from the use of an incident cohort of patients in the health administrative data and a prevalent cohort in the validation data (5.0 years in health administrative data vs 14.8 years in the validation data). However, the method of using martingale residuals to impute smoking status in health administrative data was previously demonstrated to be robust against the selection bias that affects the incidence rate of the event of interest. ${ }^{15}$ This method has not been tested in more complex situations where the association between smoking and surgery differs in the main and validation data sets. Additionally, patients in the validation data requiring surgery were younger at diagnosis than those not undergoing intestinal resection. However, there was an association between age at diagnosis and duration of follow-up in the validation data, with younger patients at diagnosis followed longer than older patients at diagnosis (A1: mean follow-up of 21 years; A2: mean follow-up of 15 years; and A3: mean follow-up of 7 years). Further, the proportion of patients who were current smokers in our validation data $(27.8 \%)$ was similar to the proportion of patients with Crohn's disease currently smoking in the province of Alberta, as estimated using the 2005 Canadian Community Health Survey (33.9\%).

We were also unable to account for phenotype or severity of asthma or Crohn's disease, which may have led to an ascertainment bias as patients with more severe IBD or asthma may have been more likely to be followed by a physician. Additionally, although we used validated algorithms to identify cases of Crohn's disease and asthma, ${ }^{17,19}$ our study may have been at risk of misclassification bias. For example, older individuals with chronic obstructive pulmonary disease may have been misclassified as asthma. Furthermore, the power and precision of our analyses were limited by the relatively low prevalence of asthma in our study population. Last, we were limited by the confounders recorded in both the health 
administrative data and our clinic-based cohort of patients, including other immune-mediated diseases, healthcare utilization practices, disease severity, medication use, and other factors that may confound the association between asthma and surgery. This limited our ability to account for further confounding variables.

\section{Conclusion}

In conclusion, we found that asthma does not alter the risk of surgery in patients with Crohn's disease. In addition, we were able to show that the adjustment for unmeasured confounding variables, such as smoking, is feasible in the context of gastrointestinal studies using health administrative data. Future studies should take a similar approach to ensure unmeasured confounding does not bias the conclusion of these studies. This method may be also applied in epidemiological studies of other associations that rely on health administrative data and have access to smaller validation samples where additional confounders are measured.

\section{Acknowledgments}

This study is based in part on data provided by Alberta Health. The interpretation and conclusions contained herein are those of the researchers and do not necessarily represent the views of the Government of Alberta. Neither the Government nor Alberta Health expresses any opinion in relation to this study. This project was funded by a Canadian Institutes of Health Research Team Grant (Health Challenges in Chronic Inflammation - Grant THC-135235).

\section{Disclosure}

Dr Kaplan was supported by a Canadian Institutes of Health Research Embedded Clinician Research Award and a Population Health Investigator Award from Alberta Innovates - Health Solutions. Dr Aviña-Zubieta is the BC Lupus Society Research Scholar and Michael Smith Foundation for Health Research Scholar. Dr Benchimol was supported by a New Investigator Award from the Canadian Institutes of Health Research, Canadian Association of Gastroenterology, and Crohn's and Colitis Canada. Dr Benchimol was also supported by the Career Enhancement Program of the Canadian Child Health Clinician Scientist Program. Dr Kuenzig was supported by a Postdoctoral Fellowship Award from the Canadian Institutes of Health Research, Canadian Association of Gastroenterology, and Crohn's and Colitis Canada. Dr Benchimol is on the editorial board for Clinical Epidemiology. The other authors report no conflicts of interest in this study.

\section{References}

1. Molodecky NA, Soon IS, Rabi DM, et al. Increasing incidence and prevalence of the inflammatory bowel diseases with time, based on systematic review. Gastroenterology. 2012;142(1):46-54.e42.

2. Masoli M, Fabian D, Holt S, Beasley R, Global Initiative for Asthma (GINA) Program. The global burden of asthma: executive summary of the GINA Dissemination Committee Report. Allergy. 2004;59(5):469-478.

3. Ng SC, Shi HY, Hamidi N, et al. Worldwide incidence and prevalence of in ammatory bowel disease in the 21 st century: a systematic review of population-based studies. Lancet. 2017: 390(10114):2769-2778.

4. Kuenzig ME, Barnabe $\mathrm{C}$, Seow $\mathrm{CH}$, et al. Asthma is associated with subsequent development of inflammatory bowel disease: a populationbased case-control study. Clin Gastroenterol Hepatol. 2017;15(9): 1405-1412.

5. Bernstein CN, Wajda A, Blanchard JF. The clustering of other chronic inflammatory diseases in inflammatory bowel disease: a populationbased study. Gastroenterology. 2005;129(3):827-836.

6. Weng X, Liu L, Barcellos LF, Allison JE, Herrinton LJ. Clustering of inflammatory bowel disease with immune mediated diseases among members of a Northern California-managed care organization. Am J Gastroenterol. 2007;102(7):1429-1435.

7. Conway G, Velonias G, Andrews E, Garber JJ, Yajnik V, Ananthakrishnan AN. The impact of co-existing immune-mediated diseases on phenotype and outcomes in inflammatory bowel diseases. Aliment Pharmacol Ther. 2017;45(6):814-823.

8. Barnes EL, Kappelman MD. Do shared exposures link the lungs and gut? Association between asthma and inflammatory bowel disease. Clin Gastroenterol Hepatol. 2017;15(9)1353-1354.

9. Frolkis AD, Dykeman J, Negrón ME, et al. Risk of surgery for inflammatory bowel diseases has decreased over time: a systematic review and meta-analysis of population-based studies. Gastroenterology. 2013;145(5):996-1006.

10. Kuenzig ME, Lee SM, Eksteen B, et al. Smoking influences the need for surgery in patients with the inflammatory bowel diseases: a systematic review and meta-analysis incorporating disease duration. $B M C$ Gastroenterol. 2016;16(1):1841.

11. Jayes L, Haslam PL, Gratziou CG, et al. SmokeHaz: systematic reviews and meta-analyses of the effects of smoking on respiratory health. Chest. 2016;150(1):164-179.

12. Benchimol EI, Manuel DG, Mojaverian N, et al. Health services utilization, specialist care, and time to diagnosis with inflammatory bowel disease in immigrants to Ontario, Canada. Inflamm Bowel Dis. 2016;22(10):2482-2490.

13. Molodecky NA, Panaccione R, Ghosh S, Barkema HW, Kaplan GG. Challenges associated with identifying the environmental determinants of the inflammatory bowel diseases. Inflamm Bowel Dis. 2011;17(8):1792-1799.

14. Ma C, Moran GW, Benchimol EI, et al. Surgical rates for Crohn's disease are decreasing: a population-based time trend analysis and validation study. Am J Gastroenterol. 2017;112(12);1840-1848.

15. Burne RM, Abrahamowicz M. Martingale residual-based method to control for confounders measured only in a validation sample in timeto-event analysis. Statist Med. 2016;35(25):4588-4606.

16. Negrón ME, Rezaie A, Barkema HW, et al. Ulcerative colitis patients with Clostridium difficile are at increased risk of death, colectomy, and postoperative complications: a population-based inception cohort study. Am J Gastroenterol. 2016;111(5):691-704.

17. Rezaie A, Quan H, Fedorak RN, Panaccione R, Hilsden RJ. Development and validation of an administrative case definition for inflammatory bowel diseases. Can J Gastroenterol. 2012;26(10):711-717.

18. Benchimol EI, Guttmann A, Griffiths AM, et al. Increasing incidence of paediatric inflammatory bowel disease in Ontario, Canada: evidence from health administrative data. Gut. 2009;58(11):1490-1497.

19. Gershon AS, Wang C, Guan J, Vasilevska-Ristovska J, Cicutto L, To T. Identifying patients with physician-diagnosed asthma in health administrative databases. Can Respir J. 2009;16(6):183-188. 
20. Wong SL, Shields M, Leatherdale S, Malaison E, Hammond D. Assessment of validity of self-reported smoking status. Health Rep. 2012;23(1):47-53.

21. Wilkins R. Version $4 E$ User's Guide: Automated Geographic Coding Based on the Statistics Canada Postal Code Conversion Files, Including Postal Codes to July 2004. Catalogue No. 93-387-XIE. Ottawa, Canada: Health Analysis and Measurement Group, Statistics Canada; 2005.

22. Plessis Du V, Beshiri R, Bollman RD, Clemenson H. Definition of "Rural." Ottawa, ON: Statistics Canada; 2002. Available from: http://www.statcan.gc.ca/pub/21-601-m/21-601-m2002061-eng.htm. Accessed November 1, 2017.

23. Silverberg MS, Satsangi J, Ahmad T, et al. Toward an integrated clinical, molecular and serological classification of inflammatory bowel disease: report of a working party of the 2005 Montreal World Congress of Gastroenterology. Can J Gastroenterol. 2005;19(Suppl A):5A-36A.

24. Therneau TM, Grambsch PM, Fleming TR. Martingale-based residuals for survival models. Biometrika. 1990;77(1):147-160.

25. Schneeweiss S. Sensitivity analysis and external adjustment for unmeasured confounders in epidemiologic database studies of therapeutics. Pharmacoepidemiol Drug Saf. 2006;15(5):291-303.

26. R Core Team. R: A language and environment for statistical computing. Available from: http://www.R-project.org/. Accessed November 1, 2017.

27. Drawing survival curves using "ggplot2" [R package survminer version 0.4.0]. 2017. Available from: https://CRAN.R-project.org/ package $=$ survminer. Accessed November 1, 2017.

28. Therneau TM. Survival analysis [R package survival version 2.41-3]. 2015. Available from: https://CRAN.R-project.org/package=survival. Accessed November 1, 2017.
29. Ditisheim S, Fournier N, Juillerat P, et al. Inflammatory articular disease in patients with inflammatory bowel disease. Inflamm Bowel Dis. 2015;21(11):2598-2604.

30. Vegh Z, Kurti Z, Gonczi L, et al. Association of extraintestinal manifestations and anaemia with disease outcomes in patients with inflammatory bowel disease. Scand J Gastroenterol. 2016;51(7):848-854.

31. Liu S, Ding J, Wang M, Zhou W, Feng M, Guan W. Clinical features of Crohn disease concomitant with ankylosing spondylitis. Medicine. 2016;95(28):e4267-e4267.

32. Dotson JL, Hyams JS, Markowitz J, et al. Extraintestinal manifestations of pediatric inflammatory bowel disease and their relation to disease type and severity. J Pediatr Gastroenterol Nutr. 2010;51(2): 140-145.

33. Orchard TR, Wordsworth BP, Jewell DP. Peripheral arthropathies in inflammatory bowel disease: their articular distribution and natural history. Gut. 1998;42(3):387-391.

34. To N, Gracie DJ, Ford AC. Systematic review with meta-analysis: the adverse effects of tobacco smoking on the natural history of Crohn's disease. Aliment Pharmacol Ther. 2016;43(5):549-561.

35. Nunes T, Etchevers MJ, García-Sánchez V, et al. Impact of smoking cessation on the clinical course of Crohn's disease under current therapeutic algorithms: a multicenter prospective study. Am J Gastroenterol. 2016;111(3):411-419.

36. Cosnes J, Beaugerie L, Carbonnel F, Gendre J-P. Smoking cessation and the course of Crohn's disease: an intervention study. Gastroenterology. 2001;120(5):1093-1099.
Clinical Epidemiology

\section{Publish your work in this journal}

Clinical Epidemiology is an international, peer-reviewed, open access, online journal focusing on disease and drug epidemiology, identification of risk factors and screening procedures to develop optimal preventative initiatives and programs. Specific topics include: diagnosis, prognosis, treatment, screening, prevention, risk factor modification,

Submit your manuscript here: https://www.dovepress.com/clinical-epidemiology-journa

\section{Dovepress}

systematic reviews, risk and safety of medical interventions, epidemiology and biostatistical methods, and evaluation of guidelines, translational medicine, health policies and economic evaluations. The manuscript management system is completely online and includes a very quick and fair peer-review system, which is all easy to use. 